\title{
Biofilm formation of Streptococcus pneumoniae from bronchial alveolar lavage and from nasal swab
}

\author{
Corresponding author: \\ Dr Agata Bialucha \\ Department of Microbiology, \\ Collegium Medicum, \\ Nicolaus Copernicus University \\ Skłodowskiej-Curie 9 , \\ 85-094 Bydgoszcz, Poland \\ Phone: (+48 52) 5854480 \\ Fax: (+48 52) 5854047 \\ E-mail: agatabialucha@wp.pl
}

Medical Research Journal 2016; Volume 1, Number 3, 100-104 10.5603/MRJ.2016.0017 Copyright ( $\odot 2016$ Via Medica ISSN 2451-2591

\begin{abstract}
Introduction. Infection induced by Streptococcus pneumoniae concerns mainly children, the elderly, and people suffering from chronic diseases. The number of deaths caused by pneumococcus infections is rising worryingly. The ability to create biofilm is the main virulence factor for S. pneumoniae. The aim of this study was to evaluate the ability to form biofilm (using two different dyes) of the bacterial strains isolated from nasal and BAL, and evaluate the relationship between antibiotic sensitivity and production of biofilm. Materials and methods. For the study of $S$. pneumoniae biofilm formation in 96-well microtitre plates, crystal violet (CV) and tetrazolium dyes - 2,3,5-triphenyl-tetrazolium chloride (TTC) were used.

Results. All isolates were able to form some degree of biofilm. The results obtained by the two methods were not significantly correlated when comparing the biofilm mass. The strains produced biofilm mass intensive when the dye was CV - 29 (96.6\%) strains of S. pneumoniae strong biofilm production. When the dye was TTC, it was observed that 14 (46.6\%) strains of $S$. pneumoniae formed strong biofilm. Conclusions. There was no significant relationship between the ability of $S$. pneumoniae to form biofilms and the source of isolates.

Key words: biofilm, crystal violet, microtitre plate, TTC, Streptococcus pneumoniae
\end{abstract}

Med Res J 2016; 1 (3): 100-104

\section{Introduction}

Streptococcus pneumoniae commonly colonises the nasopharynx asymptomatically in healthy children in the first day of life, but colonisation by the pneumococcus may occur at any time during a person's life [1]. This bacteria is the causative agent of several important diseases including pneumonia, meningitis, and otitis media [2]. Microbial factor, such as enzymes remove terminal sugars common to many human glycoconjugates, ability to acquire and disseminate resistance to multiple antibiotics, polysaccharide capsule, bacterial adhesion and biofilm formation, may be responsible for growth and survival of $S$. pneumoniae. Pneumococcal cells attaching to host cells provide more opportunities for cells for proliferation inside the human body [1] Some diseases caused by this microorganism, such as otitis and meningitis, have often been associated with the bacteria's ability to form biofilm [2]. Biofilms, are complex communities of bacteria embedded in a mature matrix composed of extracellular DNA, proteins, and polysaccharides $[1,5]$. Allegrucci et al. [6] observed that biofilm formation of $S$. pneumoniae occurred in several stages: individual planktonic cells attach to the surface, later cellular aggregates formed and created, and finally the biofilms were fully developed and attained maximum cell density (biomass) and height. Biofilm development was accompanied by increased production of several proteins involved in attachment, such as choline binding proteins (CbpA) and pneumococcal surface protein $A(\operatorname{Psp} A)$ [1]. It has been proposed that biofilm formation in vivo is intertwined with the formation of extracellular neutrophil traps [7].

Biofilm cells are difficult to eradicate with antimicrobial treatment, even when the bacterium is susceptible to antibiotics in vitro (more resistant to environmental stress). In addition, pneumococcal disease treatment bring complicated by a greater resistance to antibiotics seems to be a characteristic feature of cells that live in a sessile state (forming communities) compared to cells that adopt a planktonic lifestyle (not attached to a surface). Microorganisms in biofilms can be up to 1000 times more resistant to antibiotics than the same free-living microorganisms [2]. 
The aim of this study was to evaluate the ability to form biofilm (using two different dyes): the bacterial strains isolated from nasal and BAL, and to evaluate the relationship between antibiotic sensitivity and production of biofilm.

\section{Materials and methods}

The study included 30 S. pneumoniae strains isolated the Department of Microbiology of Dr. A. Jurasz University Hospital No 1 in Bydgoszcz, between 2009 and 2014. S. pneumoniae isolated from clinical samples such as bronchoalveolar lavage (BAL) (15 strains) and nasal swab (15 strains). The isolates were identified by standard microbiological methods, using optiochin sensitivity, yellowish/greenish discoloration of $5 \%$ sheep blood in Tryptic Soy Agar (TSA, Becton-Dickinson) plates (blood agar) around bacterial colonies, indicating incomplete haemolysis, and latex agglutination test (Slidex ${ }^{\circledR}$ pneumo-Kit, bioMérieux).

Plates were incubated at $37^{\circ} \mathrm{C}, 10 \% \mathrm{CO}_{2}$ for 18 hours. Overnight cultures of each isolate grown on blood agar were suspended in Tryptic Soy Broth (TSB, BBL) with $1 \%$ horse serum (BIOMED) and standardised to 0.5 McFarland in Tryptic Soy Broth (TSB, Becton Dickinson) with a densitometer. Each culture was incubated at $37^{\circ} \mathrm{C}, 10 \% \mathrm{CO}_{2}$ for 18 hours. The resultant cultures were harvested at 4000 RPM (Routs Per Minute) for 10 minutes, and washed with sterile, deionised water. The precipitate was suspended in TSB supplemented with $1 \%$ horse serum and standardised to $0.5 \mathrm{McF}$ arland. 96-well microtitre plates (Profilab) were filled with $200 \mu \mathrm{L}$ of TSB with horse serum, which served as negative control (six wells of microtitre plate), and $200 \mu \mathrm{L}$ of inoculum (three wells of microtitre plate). Microtitre plates were incubated in a humidity chamber at $37^{\circ} \mathrm{C}, 10 \% \mathrm{CO}_{2}$. After incubation the plates were washed three times with sterile, deionised water.

The bacterial cells that had adhered to the surface of the polystyrene were fixed by $96 \%$ ethanol $(\mathrm{POCH})$ for five minutes at room temperature after removal of alcohol, the biofilms were stained with $200 \mu \mathrm{L} 2.0 \%$ crystal violet (CV) for 15 minutes, washed with sterile, deionised water, and the plates were left to air dry for a further 15 minutes. Finally, $200 \mu \mathrm{L}$ of $96 \%$ ethanol was added to each well, and the OD (optical density) measured using a microplate reader (Synergy HT, BIO$\mathrm{TEK})$ at $570 \mathrm{~nm}\left(\mathrm{VC}_{570}\right)$.

Bacterial cells adhered to the surface of polystyrene were fixed by $100 \mu \mathrm{L} 0.1 \%$ TTC and $100 \mu \mathrm{L}$ TSB. Microtitre plates were incubated at $37^{\circ} \mathrm{C}, 10 \% \mathrm{CO}_{2}$ for two hours, and washed with sterile, deionised water. Finally, $200 \mu \mathrm{L}$ of $96 \%$ ethanol was added to each well, and the OD was measured using a microplate reader (Synergy HT) at $470 \mathrm{~nm}\left(\mathrm{TTC}_{470}\right)$.

The antibiotic sensitivity test was conducted by disc diffusion method (clarithromycin, trimethoprim-sulfamethoxazole) and E-test (ampiciline) (bioMérieux) in Muller Hinton agar (Becton-Dickinson) containing 5\% sheep's blood according to the National Reference Centre for Antimicrobial Sensitivity Testing [8]. Statistical significance was calculated by the $\chi^{2}$ test.

\section{Results}

The strains were classified as non-biofilm producers $(\mathrm{OD} \leq 0.095)$, weak biofilm producers $(0.095<\mathrm{OD} \leq 0.19)$, moderate biofilm producers $(0.19<\mathrm{OD} \leq 0.38)$, and strong biofilm producers (0.38 > OD) (Al- Martins et al., 2013). The biofilm formation by tested $S$. pneumoniae strains is presented in Table 1 and 2.

Results showed that streptococci (all strains tested) could form biofilm on the surface of polystyrene. It was observed that $14(46.7 \%)$ strains of $S$. pneumoniae produced strong biofilm, which five (33.3\%) were isolated from BAL, and nine $(60.0 \%)$ were isolated from nasal swab. The results proved that $13(43.3 \%)$ strains of $S$. pneumoniae produced moderate biofilm, of which nine $(60.0 \%)$ were isolated from BAL, and four (26.7\%) were isolated from nasal swab. It was shown that three $(10.0 \%)$ and two (13.3\%) species that produced the weakest biofilm were isolated from BAL and nasal swab, respectively.

In this study, we compared the mass of producing biofilms and susceptibility of strains representative of the four main classes of antibiotics active against $S$. pneumoniae, namely, ampicilin (for $\beta$-lactams), clarithromycin (for macrolides), and trimethoprim/sulphamethoxazole (for sulphonamide). When assessing the weight of the biofilm by means of CV, it was shown that all strains isolated from nasal swab resistant to any of the antibiotics listed above produced a strong biofilm. If the weight of the biofilm was evaluated using the TTC from five ampicillin-resistant strains, then strains 15 and 21 produced the strong biofilm, and the other three strains produced moderate biofilm (strain number: $5,8,28$ ). Strains $8,10,28$, and 29, resistant to clarithromycin, produced strong biofilm (strain numbers 10 and 29), and moderate biofilm (strain number: 8, 28 and 29). Only one strain isolated from BAL (strain number 26) resistant to trimethoprim/sulphamethoxazole poorly produced biofilm. Remaining strains resistant to sulphonamide produced strong (strain number: 6,10 , 23,29 ) and moderate biofilm (strain number $5,26,28$ ). No correlation was seen between the mass produced biofilm and resistance to amikacin, clarithromycin, trimethoprim/sulphamethoxazole. 
Table 1. Biofilm formation (OD) by $S$. pneumoniae strains $(n=30)$

\begin{tabular}{|c|c|c|c|c|c|c|c|c|c|c|c|c|c|c|c|c|}
\hline \multirow{3}{*}{$\begin{array}{l}\text { Clinical } \\
\text { material }\end{array}$} & \multirow[t]{2}{*}{ Dye } & \multicolumn{15}{|c|}{ Strain no. } \\
\hline & & 1 & 2 & 3 & 4 & 5 & 6 & 7 & 8 & 9 & 10 & 11 & 12 & 13 & 14 & 15 \\
\hline & TTC & 0.155 & 0.636 & 0.380 & 0.578 & 0.272 & 1.069 & 0.689 & 0.353 & 0.166 & 0.59 & 0.714 & 0.677 & 0.288 & 1.198 & 0.717 \\
\hline & $\mathrm{CV}_{570}$ & 0.678 & 0.643 & 1.417 & 1.192 & 0.759 & 1.15 & 1.542 & 1.02 & 1.27 & 0.624 & 2.917 & 0.954 & 1.356 & 1.754 & 1.912 \\
\hline & & \multicolumn{15}{|c|}{ Strain no. } \\
\hline \multirow[t]{3}{*}{ BAL } & & 16 & 17 & 18 & 19 & 20 & 21 & 22 & 23 & 24 & 25 & 26 & 27 & 28 & 29 & 30 \\
\hline & TTC & 0.255 & 0.338 & 0.376 & 0.668 & 0.263 & 0.494 & 0.376 & 0.687 & 0.265 & 0.296 & 0.169 & 0.36 & 0.204 & 0.511 & 0.765 \\
\hline & $\mathrm{CV}_{570}$ & 0.83 & 0.901 & 0.692 & 1.06 & 0.924 & 1.347 & 0.583 & 1.597 & 1.059 & 0.736 & 0.333 & 0.73 & 1.123 & 0.703 & 1.21 \\
\hline
\end{tabular}

$\mathrm{BAL}$ - bronchoalveolar lavage; TTC - 2,3,5-triphenyl-tetrazolium chloride; CV — crystal violet

Table 2. Interpretation of biofilm formation by $S$. pneumoniae strains $(n=30)$

\begin{tabular}{|c|c|c|c|c|c|c|c|c|c|c|c|c|c|c|c|c|}
\hline \multirow{2}{*}{$\begin{array}{l}\text { Clinical } \\
\text { material }\end{array}$} & \multirow[t]{2}{*}{ Dye } & \multicolumn{15}{|c|}{ Strain no. } \\
\hline & & 1 & 2 & 3 & 4 & 5 & 6 & 7 & 8 & 9 & 10 & 11 & 12 & 13 & 14 & 15 \\
\hline \multirow{4}{*}{$\begin{array}{l}\text { Nasal } \\
\text { swab }\end{array}$} & TTC & WBF & SBF & MBF & SBF & MBF & SBF & SBF & MBF & WBF & SBF & SBF & SBF & MBF & SBF & SBF \\
\hline & $\mathrm{CV}_{570}$ & SBF & SBF & SBF & SBF & SBF & SBF & SBF & SBF & SBF & SBF & SBF & SBF & SBF & SBF & SBF \\
\hline & & \multicolumn{15}{|c|}{ Strain no. } \\
\hline & & 16 & 17 & 18 & 19 & 20 & 21 & 22 & 23 & 24 & 25 & 26 & 27 & 28 & 29 & 30 \\
\hline \multirow[t]{2}{*}{ BAL } & TTC & MBF & MBF & MBF & SBF & MBF & SBF & MBF & SBF & MBF & MBF & WBF & MBF & MBF & SBF & SBF \\
\hline & $\mathrm{CV}_{570}$ & SBF & SBF & SBF & SBF & SBF & SBF & SBF & SBF & SBF & SBF & MBF & SBF & SBF & SBF & SBF \\
\hline
\end{tabular}

BAL — bronchoalveolar lavage; TTC - 2,3,5-triphenyl-tetrazolium chloride; CV — crystal violet; NBF — no biofilm formation; WBF — weak biofilm formation; MBF — moderate biofilm formation; SBF — strong biofilm formation

\section{Discussion}

S. pneumoniae colonises the human upper respiratory tract, and the capacity to form biofilms is believed to be important for nasopharyngeal colonisation. Asymptomatic colonisation is known to precede pneumococcal disease, for example community acquired pneumonia, because this bacteria has the ability to migrate to other niches within the human body. The presence of biofilm-like structures in the lungs of animals infected with S. pneumoniae was also documented [9]. During infection, $S$. pneumoniae exists mainly in sessile biofilms rather than in planktonic form, except during sepsis [10]. Bacteria in diverse niches may be subjected to different biotic and abiotic host factors favouring biofilm production. The present study evaluated the ability to produce the biofilm of $S$. pneumoniae strains depending on clinical origin.

Camilli et al. [11] using the colorimetric method with a dye - Hucker's crystal violet - demonstrated that five strains out of 59 were non-biofilm formers, 16 isolates could be classified as weak-biofilm formers, and 38 as strong biofilm producers. The OD values adopted in this work are slightly different from the values determined in our study, and these studies confirm that the majority of $S$. pneumoniae intensively produce biofilm.

The strong biofilm formation percentage was higher for the isolation of $S$. pneumoniae from the nasal vestibule than from the BAL, but not statistically significant $(p>0.05)$. Few authors have evaluated the ability to produce the biofilm species isolated from various clinical materials. The aim of the work by García-Castillo et al. [12] was to compare the capability for biofilm development between $S$. pneumoniae isolates from cystic fibrosis patients and from persons without cystic fibrosis, isolated from blood cultures. They observed that there were various different capabilities of biofilm formation for S. pneumoniae depending on their clinical origin. Respectively, $80.0 \%$ and $60.0 \%$ of blood isolates were able to form biofilm. Parker et al. [13], based on their own research, support the hypothesis that cells induced to form a biofilm have a greater propensity to cause pneumonia than planktonic cells. Studies by Munoz-Elisa et al. [14] showed that in the case of a commensal coloniser such as $S$. pneumoniae, these bacteria produce the virulence factors that are 
required for colonisation. Thus, strains isolated from the nasopharynx will produce biofilm. In contrast, studies by Tapianen et al. [15] and Lizcano et al. [16] claim that the ability to form biofilms in vitro could not be linked to an anatomical site from which a clinical isolate was obtained.

Currently there is no colorimetric standard method for investigating the cells in bacterial biofilms. Tetrazolium salt as TTC, 3-(4,5-dimethylthiazol-2-yl)-2,5-diphenyltetrazolium bromide (MTT), 2,3-bis-(2-methoxy-4-nitro-5-sulfophenyl)-2H-tetrazolium-5-carboxanilide (XTT), safranin, and CV are examples of dyes used to evaluate the production of biofilm. For bacteria, a common method is to quantitate the mass of biofilms by crystal violet or safranin staining. Note that when the evaluation of the ability of biofilm formation uses $\mathrm{CV}$, the dye is susceptible to inaccuracies because it is unable to distinguish between living or dead organisms, and CV binds to negatively charged molecules, such as nucleic acids and acid polysaccharides within the biofilm. TTC is reduced to red crystals of 1,3,5-triphenylformazan (TFP), which allows for the specific staining of adhered, metabolically active bacteria [17]. In this test the biofilm mass was assessed using CV and TTC, followed by extraction of bound dye with a solvent and measurement of absorption. A significant difference was demonstrated between in biofilm mass and the type of dye used in the study. The strains produced intensive biofilm mass when $\mathrm{CV}$ was used as a dye $(p<0.00001)$. The data suggests that CV gives high levels of nonspecific (false-positive) staining. Our results can be compared to studies by Sabaeifard et al. [18]. They assessed that the biofilm formed in the presence of antibiotics active against strains of Pseudomonas aeruginosa, using the TTC, XTT, and CV. Their research showed that more reproducible and accurate results can be obtained using TTC than XTT and CV. In contrast, studies by Hendiani et al. [19] showed that CV and XTT exhibit an excellent applicability for the quantification of biofilms of Acinetobacter baumannii, and the statistical analysis showed that the results from these two methods are significantly correlated. We agree with the opinion of these authors that the CV assay is cheap, and easy for the quantification of biofilms formed by microorganisms, but tetrazolium salt is more reliable and repeatable. Different type of dye used in the present study significantly affects the interpretation produce a biofilm various strains of bacteria.

Biofilm structure promotes antibiotic resistance. Reduced bacterial susceptibility to antibiotics may be associated with metabolic changes accompanying the switch from planktonic to sessile lifestyle and horizon- tal gene transfer due to the high microbial population density [20]. S. pneumoniae may be the aetiological agent of infection, complications, and death together with the emergence of strains resistant to antibiotics.

\section{Acknowledgements}

This research was financially supported by the Collegium Medicum in Bydgoszcz, Nicolaus Copernicus University in Torun with funds from the maintenance of the research potential of the Department of Microbiology.

\section{References}

1. Shak JR, Vidal JE, Klugman KP. Influence of bacterial interactions on pneumococcal colonization of the nasopharynx. Trends Microbiol 2013; 21: 129-135. https://dx.doi.org/10.1016/j.tim.2012.11.005

2. Moscoso M, García E, López R. Pneumococcal biofilms. Int Microbiol 2009; 12: 77-85. http://dx.doi.org/10.2436/20.1501.01.84

3. Weiser JN. The pneumococcus: why a commensal misbehaves. J Mol Med 2010; 88: 97-102. https://dx.doi.org/10.1007/s00109009-0557-x

4. Anderson R, Feldman C. Key virulence factors of Streptococcus pneumoniae and non-typeable Haemophilus influenzae: roles in host defence and immunisation. South Afr J Epidemiol Infect 2011; 26: 6-12. http://dx.doi.org/10.1080/10158782.2011.11441412.

5. Costerton JW, Stewart PS, Greenberg EP. Bacterial biofilms: a common cause of persistent infections. Science 1999; 284: 1318-1322. http:// dx.doi.org/10.1126/science.284.5418.1318.

6. Allegrucci M, Sauer K. Characterization of colony morphology variants isolated from Streptococcus pneumoniae biofilms. J Bacteriol 2007; 189: 2030-2038. https://dx.doi.org/10.1128/JB.01369-06.

7. Urban CF, Lourido S, Zychlinsky A. How do microbes evade neutrophil killing? Cell Microbiol 2006; 8: 1687-1696. https://dx.doi.org/10.1111/ j.1462-5822.2006.00792.x.

8. Performance standards for antimicrobial susceptibility testing; sixteenth informational supplement. CLSI M100-S16 (M2-A9 and M7-A7), January 2006.

9. Sanchez CJ, Shivshankar P, Stol K et al: The pneumococcal serine-rich repeat protein is an intra-species bacterial adhesin that promotes bacterial aggregation in vivo and in biofilms. PloS Pathog 2010; 6:8e1001044. http://dx.doi.org/10.1371/journal.ppat.1001044.

10. Trappetti C, Potter AJ, Paton AW, Oggioni MR, Paton JC. LuxS mediates iron-dependent biofilm formation, competence, and fratricide in Streptococcus pneumoniae. Infect Immun 2011; 79: 4550-4558. https://dx.doi.org/10.1128/IAl.05644-11.

11. Camilli R, Pantosti A, Baldassarri L. Contribution of serotype and genetic background to biofilm formation by Streptococcus pneumoniae. Eur J Clin Microbiol Infect Dis 2011; 30: 97-102. https://dx.doi. org/10.1007/s10096-010-1060-6.

12. García-Castillo M, Morosini MI, Valverde A et al. Differences in biofilm development and antibiotic susceptibility among Streptococcus pneumoniae isolates from cystic fibrosis samples and blood cultures. J Antimicrob Chemother 2007; 59: 301-304. https://dx.doi. org/10.1093/jac/dk/482

13. Parker D, Soong G, Planet P, Brower J, Ratner AJ, Prince A. The NanA neuraminidase of Streptococcus pneumoniae is involved in biofilm formation. Infect Immun 2009; 77: 3722-3730. https://dx.doi. org/10.1128/IAI.00228-09.

14. Munoz-Elias EJ, Marcano J, Camilli A. Isolation of Streptococcus pneumoniae biofilm mutants and their characterization during nasopharyngeal colonization. Infect Immun 2008; 76: 5049-5061. https:// dx.doi.org/10.1128\%2FIAl.00425-08

15. Tapiainen T, Kujala T, Kaijalainen T et al. Biofilm formation by Streptococcus pneumoniae isolates from paediatric patients. APMIS 2010; 118: 255-260. https://dx.doi.org/10.1111/j.1600-0463.2010.02587.x.

16. Lizcano A, Chin T, Sauer K, Tuomanen El, Orihuela CJ. Early biofilm formation on microtiter plates is not correlated with the invasive disease 
potential of Streptococcus pneumoniae. Microb Pathog 2010; 48 : 124-130. https://dx.doi.org/10.1016/j.micpath.2010.01.002

17. Brown $\mathrm{HL}$, van Vliet $\mathrm{AH}$, Betts RP, Reuter M. Tetrazolium reduction allows assessment of biofilm formation by Campylobacter jejuni in a food matrix model. J Appl Microbiol 2013; 115: 1212-1221. https:// dx.doi.org/10.1111/jam.12316.

18. Sabaeifard P, Abdi-Ali A, Soudi MR, Dinarvand R. Optimization of tetrazolium salt assay for Pseudomonas aeruginosa biofilm using microtiter plate method. J Microbiol Methods 2014; 105: 134-140. https://dx.doi.org/10.1016/j.mimet.2014.07.024

19. Hendiani S, Abdi-Ali A, Mohammadi P. Comparison of two methods for quantification of Acinetobacter baumannii biofilm formation. BJM 2014; 2: 51-56.

20. Bueno J. Anti-biofilm drug susceptibility testing methods: looking for new strategies against resistance mechanism. J Microbial Biochem Technol 2014; S3, article 004. http://dx.doi.org/10.4172/1948-5948.S3-004. 